\title{
Evaluación del sistema de reutilización y disposición final de residuos generados por la empresa BELCORP S.A.
}

\author{
Evaluation of the reuse and final disposal system of residues generated by the company BELCORP \\ S.A.
}

\author{
Ángela Ascarza A. ${ }^{1}$; Víctor Miyashiro K. ${ }^{2}$
}

\begin{abstract}
Resumen
El presente estudio mide las posibilidades de aplicación de un sistema de reutilización y cambio de tecnología de disposición final de residuos sólidos, con la finalidad de contribuir a la solución del problema de acumulación de inventarios de productos obsoletos, de la Corporación Internacional BELCORP. Se realizó un diagnóstico inicial de los procesos que generan residuos sólidos en la empresa y se ejecutó la caracterización del tipo de resina de los materiales plásticos. Además, se evaluó el mercado de empresas recicladoras y fabricadoras de materiales plásticos y se planteó el análisis de una nueva tecnología de disposición de residuos sólidos que mejorara dicho proceso en la empresa. Finalmente, se elaboró un análisis previo de variables económicas con los datos obtenidos en pasos anteriores. Los resultados se sintetizan en valores monetarios que comprueban la viabilidad económica tanto del sistema de reutilización de residuos sólidos plásticos planteado, como de la propuesta de utilización de la incineración controlada como nueva tecnología de disposición final de residuos sólidos no reutilizados.
\end{abstract}

Palabras clave: residuos sólidos; plásticos; reutilización; reciclaje; incineración controlada.

\begin{abstract}
This study evaluates the applicability of a reuse system and change in technology of final disposal of solid residues, with the objective of contributing to the solution of the obsolete products inventory accumulation of the International Corporation BELCORP. We performed an initial diagnosis of the processes involved in solid waste generation in the company and performed the characterization of the resin type used in plastic materials. We also assessed the market of recycling companies and plastic production, and established the analysis of a new technology of solid waste disposal to improve the current process in the company. Finally, we developed a preliminary analysis of economic variables with data obtained in the previous steps. The results are summarized in the monetary values that prove the economic viability of both the plastic solid waste reuse system and the proposed use of Controlled Incineration as a new technology for the final disposal of non-reused solid waste.
\end{abstract}

Keywords: solid waste; plastics; reuse; recycling; controlled incineration.

\section{Introducción}

La generación y acumulación desmesurada de residuos provenientes de los diferentes tipos de industrias es uno de los temas en materia ambiental que ha tomado importancia en todo el mundo. La proliferación de industrias en el mercado actual ha aumentado la generación de residuos exponencialmente, lo que causa graves problemas ambientales, sociales y económicos. Es por ello que toda industria debe disponer de manera adecuada sus residuos y, más aún, debe adoptar un adecuado manejo de los mismos, intentando reducir, reusar y reciclar en la medida que les sea posible.

Esta situación se torna más compleja si se conoce que la mayoría de industrias no pueden reducir sus residuos debido a que dependen completamente del mercado, si una empresa no se adapta al mercado no sobrevive en él. En el caso nacional, una empresa generalmente no busca aplicar alguna estrategia de minimización y control de sus residuos, sino que se conforma con deshacerse de los mismos por medio de alguna compañía de disposición final que asegure la rápida, pero quizás no adecuada, forma de eliminarlos.

El presente estudio evalúa la aplicación de un sistema de reutilización y disposición final de los residuos sólidos de la empresa BELCORP S.A., dueña de las firmas L'bel, Ésika y CyZone, especialistas en productos de belleza para mujeres. Los resultados podrán servir de modelo a otras empresas de características similares que estén en la búsqueda de soluciones eficientes para la reducción de sus residuos industriales, desde una perspectiva ambiental y

Ingeniera Ambiental. Universidad Nacional Agraria La Molina, Lima, Perú.

2 Profesor Principal, Departamento Académico de Ingeniería Ambiental, Física y Meteorología,

Facultad de Ciencias. Universidad Nacional Agraria La Molina, Lima, Perú. E-mail: vmk@lamolina.edu.pe 
económicamente sostenible (Ayres y Ares, 2004; Cervantes et al., 2009).

\section{Materiales y métodos}

BELCORP es una corporación internacional fundada en el Perú hace más de 40 años, es dueña de firmas de cosméticos, productos de cuidado personal, moda, hogar y accesorios; estas son L'bel, Ésika y CyZone. Actualmente, posee dos plantas principales de producción, una ubicada en Lima (Perú) y la otra en Bogotá (Colombia); la de Perú es subcontratada por la empresa YOBEL. El estudio se realizó tanto en las instalaciones de la casa matriz de BELCORP, que se ubica en la Avenida Canaval y Moreyra 480, San Isidro, como en su planta de producción que se sitúa en la Avenida San Andrés 150, Los Olivos (zona industrial), ambas en la provincia y departamento de Lima. La investigación se realizó en cinco fases:

\section{Diagnóstico inicial de la empresa BELCORP}

Se describió cada actividad involucrada con los residuos sólidos dentro de la empresa y también a los actores principales que intervinieron en la generación de los mismos.

Esta información se obtuvo por medio de visitas a la casa matriz de BELCORP y a la planta de producción donde la empresa YOBEL se encarga de la fabricación de los productos comercializados por BELCORP. Durante estas visitas, se recopiló información por medio de reuniones con los responsables del suministro de materiales, producción y eliminación de residuos, lográndose conocer a detalle el funcionamiento de estas actividades principales.

Para lograr una comprensión total de la data brindada por estas empresas, se aprendió a utilizar el software Solutions Applications and Services (SAP), el cual sirve para el almacenamiento y manejo de data en ambas empresas (BELCORP y YOBEL).

\section{Caracterización del tipo de resina plástica}

Se determinó los diversos tipos de resinas con que fueron elaborados los residuos sólidos plásticos (RSP) de la empresa, con la finalidad de analizar la viabilidad técnica para ser reciclados (Lorenzo, 2008; Vargas, 2004; Laguna, 1993; Matthes, 1991).

Las actividades realizadas en el desarrollo de la caracterización fueron las siguientes:

- Selección de muestras de RSP, haciendo uso del software SAP.

- Aplicación de pruebas físicas a las muestras seleccionadas, como quemado, trituración e inmersión en agua.

\section{Análisis del mercado de reciclaje y fabricación de productos plásticos}

Se realizó la búsqueda de empresas especialistas en reciclaje y fabricación de plástico en el mercado local que trabajaran con los diferentes tipos de resinas encontrados. Una vez contactadas y seleccionadas, se organizaron visitas para comprobar su capacidad de trabajo con grandes volúmenes de RSP. Las visitas consistieron en reuniones con los jefes de comercio y producción de cada empresa para evaluar las cotizaciones por los servicios a prestar: reciclaje y fabricación de materiales plásticos.

\section{Análisis preliminar de variables económicas para la reutilización de residuos sólidos plásticos}

La evaluación económica realizada compila datos obtenidos en pasos anteriores para utilizarlos en una relación simple de costo y beneficio, la cual se realizó por medio de situaciones supuestas que permitieron el desarrollo del análisis preliminar de variables económicas. Los supuestos involucrados se dieron tomando como base la fabricación de un producto terminado de la empresa, el cual fue el desodorante llamado "Mercy" de la marca Ésika. Los escenarios planteados fueron:

- Escenario A: Fabricación de producto haciendo uso de un envase con material reciclado.

- Escenario B: Fabricación del mismo producto usando envase de materia prima virgen.

La fórmula usada en la relación costo-beneficio fue:

$\mathbf{B T}=\mathbf{I T}-\mathbf{C T}$

Donde:

$\mathrm{BT}=$ Beneficio Total

$\mathrm{IT}=$ Ingreso Total (ventas)

$\mathrm{CT}=$ Costo Total (gastos fabricación)

\section{Análisis de la propuesta tecnológica de disposición final de residuos sólidos}

Se evaluó la tecnología de incineración controlada propuesta a BELCORP para ser usada en el proceso de disposición final de sus residuos sólidos, con la finalidad de comprobar si es conveniente para la empresa en términos económicos y ambientales (Terán et al., 2008; Kaynak y Topal, 2005; Allsopp et al., 2004)

Para la evaluación se realizaron reuniones con el representante de ventas en Perú del Mid West International Group - MWIG (2004), empresa estadounidense que actualmente posee cuatro plantas de incineración controlada en Perú para la disposición final de residuos municipales e industriales. Los principales puntos considerados en este análisis fueron los siguientes:

- Variables económicas, como costos de servicio, transporte y otros gastos.

- Peso total de residuos sólidos a disponer en un año por cada tecnología.

En la evaluación del cuidado ambiental se elaboró un cuadro resumen en el que se describen cada uno de los criterios tomados por la empresa MWIG en el control y protección del ambiente (Aversano, 2006).

\section{Resultados y discusión}

Del diagnóstico se obtuvo que la empresa BELCORP cuenta en con S/. 1.513.727,48 en envases plásticos no aptos para la venta y que pasan al proceso de disposición final, estos son útiles para la propuesta del sistema de reutilización de RSP. En la Fig. 1 se puede observar que, en proporción, esta cantidad abarca el 47,42 \% del total; mientras que el total en peso, que representa al conjunto de 
RSP utilizados como material disponible para el reciclaje y reutilización, es de $38.952,12 \mathrm{~kg}$.

La caracterización del tipo de resina plástica se muestra en la Fig. 2.

De la Figura anterior se observa que el $89 \%$ de las muestras

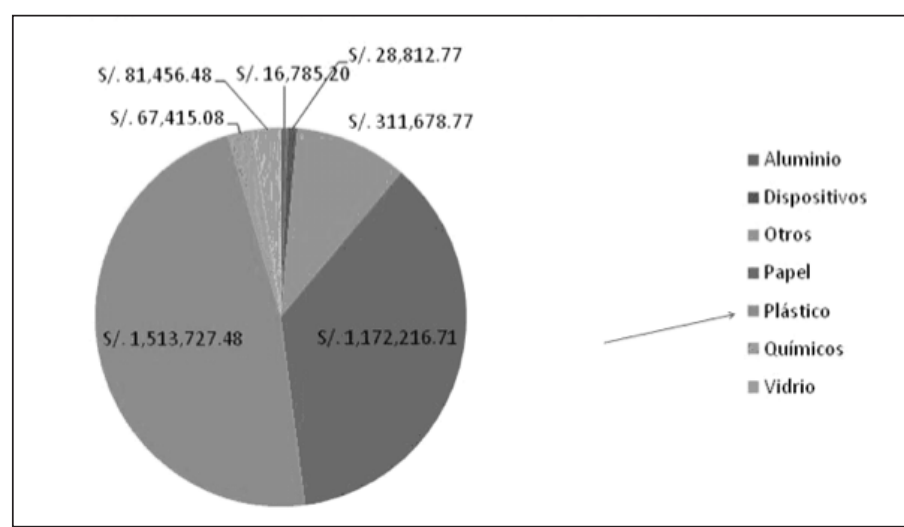

Figura 1. Inventario de envases plásticos obsoletos, al $22 / 02 / 10$

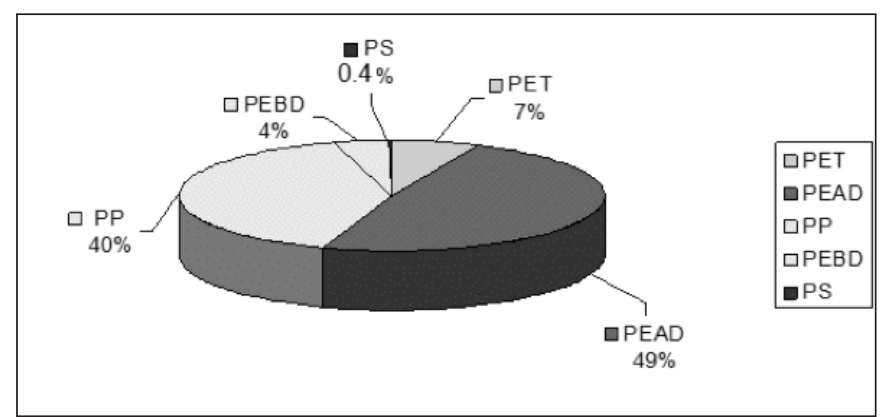

Figura 2. Tipo de resina plástica presente en los RSP

analizadas son del tipo PEAD y PP, las cuales provienen de envases de productos como champú, acondicionadores y desodorantes; mientras que la minoría (11\%) es de resina tipo PET, PEAB y PS, usada en pequeños productos como frascos de muestras de perfumes, tubillos dispersores y frascos de perfumes, respectivamente.

De las resinas identificadas, las que se utilizaron en la evaluación del sistema de reutilización fueron las del tipo PEAD y PP, que son fácilmente reutilizables y conocidas en el mercado del reciclaje. La empresa identificada para la evaluación del proceso de reciclaje fue Argu’s Service S.R.L. En la Tabla 1, se describen los principales alcances del servicio que brinda esta empresa.

Para el servicio de fabricación de envases plásticos, la

Tabla 1. Datos obtenidos de la empresa Argu's Service S.R.L

\begin{tabular}{|l|l|}
\hline $\begin{array}{l}\text { Capacidad de } \\
\text { trabajo }\end{array}$ & $\begin{array}{l}10 \text { toneladas (por ciclo de } \\
\text { producción) }\end{array}$ \\
\hline $\begin{array}{l}\text { Ubicación de } \\
\text { la planta }\end{array}$ & $\begin{array}{l}\text { Mz A Lt } 3 \text { Calle San Carlos - San } \\
\text { Anita }\end{array}$ \\
\hline $\begin{array}{l}\text { Características } \\
\text { del servicio }\end{array}$ & $\begin{array}{l}\text { Incluye pelletizado, con entrega de la } \\
\text { materia prima reciclada dividida por } \\
\text { calidad de resina y colores. }\end{array}$ \\
\hline Costo total & S/. 0,833 por kg incluido IGV \\
\hline
\end{tabular}

Tabla 2 presenta los datos obtenidos para cada empresa identificada.

De la Tabla anterior, el dato utilizado en la evaluación

Tabla 2. Datos porcentuales del costo total de fabricación

\begin{tabular}{||c|c|c|c|}
\hline \multicolumn{1}{|c|}{ Empresa } & $\begin{array}{c}\text { \% Mano } \\
\text { de obra }\end{array}$ & $\begin{array}{c}\text { \% Materia } \\
\text { prima }\end{array}$ & $\begin{array}{c}\text { Promedio final } \\
\text { Materia Prima } \\
\text { (\%) }\end{array}$ \\
\hline Solasky S.A.C & 62 & 38 & \multirow{2}{*}{40} \\
\cline { 1 - 2 } \begin{tabular}{|} 
In d u s tri a s \\
Niko S.A.
\end{tabular} & 58 & 42 & \\
\hline AE1 S.A. & 60 & 40 & \\
\hline
\end{tabular}

del sistema de reutilización fue el de costo porcentual en adquisición de materia prima, puesto que es el porcentaje que no se pagaría al contratar el servicio por contar con materia prima proveniente del proceso de reciclado de los RSP de la empresa BELCORP. Usando el promedio calculado con los tres datos, resulta el $40 \%$ y el costo total de la fabricación de los envases plásticos es solo del $60 \%$ del real.

Del análisis preliminar de las variables económicas del sistema de reutilización de residuos sólidos plásticos se obtuvo la Tabla 3, como resultado para el escenario A (fabricación del desodorante Mercy con envases de material reciclado).

El costo total de la fabricación de un desodorante con envase

Tabla 3. Costos en el escenario A

\begin{tabular}{|c|c|c|}
\hline Descripción & Cantidad & $\begin{array}{c}\text { Costo } \\
\text { acumulado } \\
(\mathrm{S} / .)\end{array}$ \\
\hline $\begin{array}{lll}\begin{array}{l}\text { Inventario } \\
\text { disponibles }\end{array} & \text { de } & \text { RSP } \\
\end{array}$ & $38.952,120 \mathrm{~kg}$ & $1.513 .727,48$ \\
\hline $\begin{array}{l}\text { RSP usados } \quad(89 \% \quad \text { - } \\
\text { PEAD y PP })\end{array}$ & $34.667,387 \mathrm{~kg}$ & $1.513 .727,48$ \\
\hline $\begin{array}{l}\text { Transformación } \\
\text { materia prima (menos } 3 \\
\% \text { por pérdidas durante } \\
\text { el proceso) }\end{array}$ & $33.627,365 \mathrm{~kg}$ & $1.542 .605,41$ \\
\hline $\begin{array}{l}\text { Fabricación de envases, } \\
\text { tapa más frasco (Incluido } \\
\text { el } 40 \% \text { menos por } \\
\text { materia prima) }\end{array}$ & 672.547 unid. & $333.072,30$ \\
\hline $\begin{array}{l}\text { Valor de los envases } \\
\text { fabricados }\end{array}$ & 672.547 unid. & $1.875 .677,70^{(1)}$ \\
\hline $\begin{array}{l}\text { Valor del desodorante } \\
\text { con envase reciclado }\end{array}$ & 672.547 unid. & $5.680 .950,40^{(2)}$ \\
\hline
\end{tabular}

Nota: (1) Valor acumulado, suma de los dos últimos valores. (2) Valor acumulado, suma del último valor más el del costo aparte de fabricación del contenido del producto.

de material reciclado, alcanza los S/. 5.680.950,4 nuevos soles, el cual incluye el costo de reciclado, fabricación y el gasto en la adquisición de los RSP utilizados como materia prima no virgen para los envases, por la empresa BELCORP. A este valor se le restó los gastos que la 
empresa deja de efectuar, por dar movimiento al inventario obsoleto que viene acumulando en los almacenes, el cual básicamente se da por mantenimiento, almacenaje y disposición final.

El valor final del costo total (CT) para el escenario A fue de S/. 3.991.774,3, mientras que el valor para los ingresos totales (IT) se obtuvo de la venta del total de 672.547 unidades fabricadas, por el costo en el mercado del desodorante, el cual alcanza los S/. 10.760.756,9. Con la aplicación de la fórmula de relación costo-beneficio, el total llega a S/. 6.768.982,6, valor final para los beneficios totales (BT) obtenidos en el análisis del escenario A.

La Tabla 4 indica el CT de la fabricación del mismo desodorante, pero en el Escenario B.

De la Tabla anterior se obtiene que la empresa BELCORP

Tabla 4. Costos en el Escenario B

\begin{tabular}{|l|c|c|}
\hline \multicolumn{1}{|c|}{ Producto } & $\begin{array}{c}\text { Cantidad usada } \\
\text { por unidad }\end{array}$ & $\begin{array}{c}\text { Costo Unitario } \\
\text { (S/.) }\end{array}$ \\
\hline Bulk & 54,5 gr. & 5,450 \\
\hline Esfera rollon & 1 unid. & 0,208 \\
\hline $\begin{array}{l}\text { Envase (Tapa y } \\
\text { frasco) }\end{array}$ & 1 unid. & 0,826 \\
\hline $\begin{array}{l}\text { Total por unid. } \\
\text { de desodorante }\end{array}$ & & $\mathbf{6 , 4 8 0}$ \\
\hline
\end{tabular}

gastó S/. 6,48 en la fabricación de una unidad del desodorante "Mercy", por lo que el CT de la fabricación de los 672.547 desodorantes (siguiendo la cantidad usada en el escenario anterior) fue de S/.4.358.104,56. Mientras que el valor para el IT viene a ser de igual forma que el caso anterior, S/. 10.760.756,90. Obteniéndose finalmente para el valor del BT, aplicando la fórmula, S/. 6.402.652,34 en el Escenario b.

Comparando ambos resultados de BT, el escenario de reutilización de RSP es mayor que el otro, comprobándose que la empresa puede transformar su inventario obsoleto con nuevos productos que puedan ser vendidos en el mercado, generando mayor beneficio económico que manteniéndolo en almacenes y llevándolo a disposición final.

En la evaluación económica de la propuesta del cambio de tecnología de disposición final, se obtuvo la Tabla 5, en el cual se muestra la comparación de Costos finales de disposición final anual, para la tecnología de relleno

Tabla 5. Cuadro comparativo de costos totales disposición final

\begin{tabular}{|l|r|c|}
\hline & $\begin{array}{c}\text { Costo rellenos } \\
\text { (S/.) }\end{array}$ & $\begin{array}{c}\text { Costo } \\
\text { incineración } \\
\text { controlada } \\
\text { (S/.) }\end{array}$ \\
\hline Residuos No Peligrosos & $57.899,00$ & 47.918 \\
\hline Residuos Peligrosos & $68.922,00$ & 16.222 \\
\hline Adicionales & $5.208,00$ & 17.817 \\
\hline Total & $\mathbf{1 3 2 . 0 2 9 , 2 4}$ & $\mathbf{8 1 . 9 5 7}$ \\
\hline
\end{tabular}

sanitario - seguridad, que es la que actualmente usa la empresa y la propuesta de incineración controlada.

De acuerdo con la Tabla anterior, la tecnología de incineración controlada es menos costosa para la empresa BELCORP, debido a que reduce los costos de disposición final en $37,93 \%$, lo que corrobora que la nueva tecnología propuesta es viablemente económica para la empresa. Por otro lado, en el análisis ambiental de la tecnología propuesta, se procedió a identificar las principales medidas de disminución de emisiones, recuperación de energía, generación de sub-productos y otras características del servicio brindado por la empresa incineradora que aseguran el cuidado del ambiente. Estos se muestran en la Tabla 6.

El resultado de la evaluación comprueba que la utilización

Tabla 6. Principales medidas de protección ambiental tomadas por la empresa MWIG

\begin{tabular}{|ll|}
\hline \multicolumn{1}{|c|}{ Medidas de protección ambiental } \\
\hline 1. & Uso de una extensión de terreno no mayor a 5 hectáreas. \\
\hline 2. & $\begin{array}{l}\text { Abatimiento de emisiones de hasta el } 95 \% \text { de los } \\
\text { contaminantes generados. }\end{array}$ \\
\hline 3. & $\begin{array}{l}\text { Cumplimiento de las normativas legales de emisiones de } \\
\text { gases y partículas por incineración de residuos basado en } \\
\text { los criterios dados por la Agencia de Protección Ambiental } \\
\text { (EPA). }\end{array}$ \\
\hline 4. & $\begin{array}{l}\text { Funcionamiento energéticamente autosuficiente de la } \\
\text { planta incineradora. }\end{array}$ \\
\hline 5. & $\begin{array}{l}\text { Generación de sub-productos que son brindado a la } \\
\text { sociedad. }\end{array}$ \\
\hline 6. & Expedición de un certificado ambiental para el cliente. \\
\hline
\end{tabular}

de la tecnología de incineración controlada beneficia económicamente a la empresa BELCORP y procura el cuidado ambiental con las medidas de disminución de la contaminación.

\section{Conclusiones}

Se identificó que los problemas de acumulación de residuos de BELCORP no pueden evitarse totalmente, pero sí remediarse y minimizarse con la aplicación del sistema de reutilización y cambio de tecnología de disposición final propuestos. La empresa cuenta con 38,9 toneladas RSP como material útil para la aplicación del sistema de reutilización propuesto.

El $89 \%$ del inventario plástico es del tipo PEAD y PP, que son resinas útiles para el reciclaje y la producción de nuevos productos que pueden ser vendidos en el mercado. BELCORP tendría que pagar S/. 0,833 por $\mathrm{kg}$ de RSP llevado a materia prima no virgen y solo el $60 \%$ del costo de fabricación de productos plásticos, proporcionando la materia prima para el proceso.

Se dejaría de perder hasta S/. 366.330,3 anuales con la aplicación del sistema de reutilización de RSP propuesto debido a que sus gastos se reducirían en comparación con lo que se aplica actualmente.

La tecnología de disposición final propuesta demuestra ser económicamente beneficiosa para la empresa, pues reduce el costo de disposición final en $37,9 \%$, además de brindarle un servicio que le asegure el cuidado ambiental en el proceso de disposición. 


\section{Literatura citada}

Allsopp, M.; Costner, P. y Johnston, P. 2004. Estado del Conocimiento de los Impactos de los Incineradores de Residuos en la Salud Humana. Reino Unido: Laboratorios de Investigación de la Universidad de Exeter, pp. 4-10.

Aversano, Nicolás. 2006. El Calentamiento Global: Bonos de Carbono, una alternativa. Modelización y Simulación de Sistemas Económicos. España, pp. 10- 19.

Ayres, R. y Ayres, I. 2001. A Handbook for Industrial Ecology. (2da. edición). Edward Elgar, versión en español. Northampton, pp. 20-31.

Cervantes Torre, M.; Sosa Granados, R.; Rodríguez Herrera, G. y Robles Martínez, F. 2009. Ecología industrial y desarrollo sustentable. Revista Académica de la FI-UADY. México. 63-66.

Kaynak, B. y Topal, H. 2005. Peach and apricot stone combustion in a bubbling fluidized bed. Fuel Processing Technology, versión traducida al español, pp. 1175-1193.

Laguna, O. 1993. Reutilización de Residuos de plásticos. Análisis e Identificación de residuos urbanos. Revista de Plásticos Modernos FOCITEC, 330:125-136.

Lorenzo, H. 2008. Aplicación de nuevas tecnologías en la realización de herramientas para la fabricación de plásticos. Madrid, España: Universidad Politécnica de Madrid, Departamento de Ingeniería Mecánica y de Fabricación, pp.16-30.

Matthes, G. 1991. Reciclaje de materiales plástico. Plásticos universales. Colombia, pp. 111-114. ISSN 03034011.

Mid West International Group. 2004. Administración, manejo, aprovechamiento y transformación de desechos municipales, industriales, domésticos, comerciales y patológicos, pp. 3 -28. Miami, Estados Unidos.

Terán Saucedo, R.A.; López Ocaña, G.; Bautista Margulis, R. G y Rubio Arias, H.O. 2008. Combustión de Residuos Sólidos en un sistema de Lecho Fluidizado experimental, pp. 89-100. México: Universidad Juárez Autónoma de Tabasco. ISSN 0186-2979.

Vargas F., Luis. 2004. Reciclado de Plásticos. Red Panamericana de Información en Salud Ambiental, CEPIS, pp. $125-127$ 